\title{
Exploratory factorial structure of the experience induced abortion
} Cruz García-Lirios $^{{ }^{*} \text {, Sonia Sujell Velez-Baez }}{ }^{2}$, Sofia López de Nava-Tapía ${ }^{3}$

*Corresponding Author: Cruz García-Lirios

Received date: April 01, 2021; Accepted date: July 02, 2021; Published date: July 08, 2021

Citation: Cruz García-Lirios, Sonia Sujell Velez-Baez, Sofia López de Nava-Tapía (2021) Exploratory factorial structure of the experience induced abortion. J. Neuroscience and Neurological Surgery. 9(2); DOI:10.31579/2578-8868/174

Copyright: ( 2021 Cruz García-Lirios, This is an open-access article distributed under the terms of The Creative Commons Attribution License, which permits unrestricted use, distribution, and reproduction in any medium, provided the original author and source are credited

\begin{abstract}
:
From a review of studies on abortion, a non - random selection of 100 college students to reliability and validity eight subscales of norms, beliefs, values, perceptions, attitudes, motives, intentions and experiences concerning the request was made of assisted legal abortion. The results show that family standards affect experiences, but intentions and perceptions also impacted on them. In this regard, it is noted on the influence of more than rational support when the request for abortion, attend lectures on the subject, religious talks, consultations with experts, legal assistance procedures or curettage promoting affective processes. Such an exercise will allow to anticipate the effects of sexual health programs on student groups and the use of contraceptives.

Key words: values; norms; beliefs; perceptions; motives; attitudes; intentions; behaviors
\end{abstract}

\section{Introduction}

The objective of the present work lies in the contrast of a model of determining trajectories of the experiences of legally assisted interruption of pregnancy in students.

From a literature review that highlights: 1) the type of relationship and the expectations of it as determinants of the decision to abort or procreate; 2) the economic stability of the couple and 3) employment opportunities as the determinants of the experiences of interruption of pregnancy, we proceed to contrast the relationships between the factors involved (Calderón \& Alzamora, 2009).

Induced or assisted abortion is defined as the request to terminate pregnancy due to poverty, risk or lack of skill. The curettage is the main method of abortion, although the legal marital commitment inhibits application. In this sense, relationships without commitment encourage assisted interruption and the relegation of the couple. It is also related to school discrimination in adolescent groups, mainly when the pregnancy was due to lack of contraception and generates a negative coping style. However, the risk of assisted interruption is more linked to medical malpractice. When prescribing the contraceptive pill, physicians obviate latent risks due to low effectiveness (Chávez \& Zapata, 2009).

The problem is circumscribed to the assisted interruption of pregnancy because of the lack of reproductive and sexual health. Emotional chains are the relationships of dependence between values, norms, beliefs, perceptions, motives, attitudes, intentions and behaviors related to the request of abortion more than rational links (Fernandez, Carr, Leal, Carrillo, Carrillo, Lozano, Fernandez \& Pastor, 2010).
The assisted interruption of pregnancy has been explained from socioeconomic and demographic factors. Based on sex differences, age, schooling. However, norms, values, representations, perceptions, knowledge, beliefs, attitudes, decision and contraception have been modeled as independent variables of assisted abortion or posttraumatic stress of curettage (Galváo, Díaz, Osis, lark \& Ellerston, 2000).

The answer to such question is in assumptions according to which a behavior associated with the assisted interruption of pregnancy is determined by combinations of relationships between values, beliefs, norms, perceptions, attitudes, motives and intentions (González, 2000).

In the case of values in the choice of couple, the type of relationship and decision making were determinants of the experiences of legally assisted interruption of pregnancy, but beliefs about the quality of life and family welfare inhibited an application curettage

(Obeichina, Mbamara, Uboataja, Ogelle \& Akabuike, 2010).

It is true that the autonomic formation of women determined a consensual choice, but the absence of a formal education inhibited the request for curettage, expanding the family influence, mainly the incidence of the mother in the decision of abortion (Oduwole, 2010).

Although, the expectations of choice of couple influenced through the coexistence the request of curettage, it is the degree of perceived commitment of the relationship that determined the knowledge and the documentation of the legally assisted interruption of the pregnancy (Olaitan, 2011). 
In this way, the cumulative intentions of requesting a curettage on the part of the woman, her family and her partner do not seem to inhibit the expectation of procreating a family as long as the economic situation is perceived as favorable. In contrast, when a woman has the resources for procreation, she reduces her intentionality to interrupt pregnancy (Petracci, 2011).

Therefore, the specification of a model is limited to the values, norms, perceptions, beliefs, attitudes, knowledge, intentions and experiences around the request of interruption of pregnancy (Piaroza, Kazembe, Maluwa, Hirwa \& Chimango, 2012).

However, unlike classical structural studies in which values and norms were considered sociocultural determinants of sociocognitive factors such as beliefs, attitudes, perceptions, knowledge and intentions, these in turn behavioral variables Like the requests and the self-care for the interruption of the abortion, now the socio-cultural variables are related in such a way that they can establish direct relationships with the behavior (Ramírez, 2000).

In fact, in the midst of which these sociocultural variables are specified, they determine, to a greater extent, the behavioral variables such as the norms of self-care that affect the treatment's treatment, or the autonomic values that determine the intentions of procreation or family planning (Rodríguez \& Mayol, 2011).

This is how, to the extent that the estimating instruments of the variables have specialized, they are able to establish direct relationships between certain types of norms and values with respect to sociocognitive variables, but also in relation to behavioral variables (Salazar, 2007).

Nowadays it is known that insofar as the instruments measure specific situations of value judgments or follow-up of norms, they will more accurately predict the specific actions in those situations, contexts and specific scenarios that force a greater number of decisions to be made, seeking greater efficiency (Serrano, 2011).

In the case of the legally assisted interruption of pregnancy it has been observed that it is not always a decision that favors the woman or harms her completely, granting the opportunity to amend the error later as is the case of procreating to receive support social and state, or, abort to receive social recognition or the group to which you belong or want to lose (Shelat, Hihoriya \& Kumbar, 2012).

This is how the advance of the legally assisted abortion law has led to decisions that do not always underlie a reflection of the expected benefit of women, but also not of their effects on their economy, relationships or emotional situation, but rather on the social benefits implied by procreation or abortion depending on the progressive or traditional sector to which one belongs (Silva, Ashton \& McNeil, 2011).

Even the diversification of these groups has generated various decision options in which abortion or procreation generates recognitions from different socioeconomic strata that are willing to support the decision, whatever it may be provided that it conforms to its norms and values (Sultan \& Malik, 2010).

In this way, norms and values interacting with sociocognitive factors generate a structure of intentionalities that will determine the request for interruption of pregnancy or procreation. The emotional and decisional intelligence of the woman in this scenario is fundamental to establish criteria of choice from the first couple with whom she will have sexual relations (Tapia, Villaseñor \& Nuño, 2008).

A greater formal instruction of knowledge and skills in the choice of sexual partners is a fundamental determinant of the request for interruption of pregnancy and procreation, depending on the interrelation with traditional or progressive groups (Távara \& Sacsa, 2008).

Therefore, it is necessary to relate sociocultural variables such as values and norms with behavioral variables, as well as sociocognitive variables as determinants of actions in specific contexts and in immediate decisionmaking situations with short- and medium-term implications (Viveros \& Navia, 2012).

\section{Method}

Design. A correlational and transversal study was carried out.

Shows. A non-probabilistic selection was made of 100 students from a public university in Mexico (45\% men and 65\% women, $M=19.21, S D$ $=0.81$ for age and $M=718$ USD, $S D=1.92$ USD). For monthly family economic income).

Instrument. Scale of Induced Abortion (SIA-24), It includes 23 statements about situations of values, norms, beliefs, perceptions, attitudes, knowledge and intentions regarding the legally assisted interruption of abortion. Each is answered with one of the options ranging from $0=$ "not likely" to 5 = "quite likely".

In the case of values and norms refer to contexts delimited around the type of partner relationship with respect to the choice of procreation or abortion: "I will choose procreation if my income allows the maintenance", or: "I will always choose what that can contribute when having a son maintenance".

The perceptions, beliefs, attitudes, knowledge and intentions were built based on criteria situations of choice of costs and benefits in specific scenarios: "I will choose procreation only if I can give a ", or: "Procreation is a choice of maintenance "...

In relation to the behavioral variables, dilemmas of choice were specified regarding the effects of procreation or the interruption of pregnancy: "I will request an abortion if my economic situation is favorable", "I will procreate if my situation is uncertain"

Procedure. Aron was carried out preliminary interviews to explore norms, values, perceptions, beliefs, attitudes, motives, intentions and actions regarding the request for induced abortion. Once the conceptual dimensions were established, the items were constructed. Subsequently, it was proceeded to its massive application and exclusion of the items. Once the subscales were established, they were finally applied. When the questionnaires were delivered, they were informed that their answers would not indirectly or directly, negatively or positively affect their qualifications. They were informed that they would have only 25 minutes to answer the term which would be collected without exception questionnaires. The questionnaires that presented the same response or the absence of them were excluded from the capture. Subsequently, data were processed in the Statistical Package for Social Sciences (SPSS and AMOS version 20,0).

Analysis. A dependency relationship between each of the variables following the established hypothesis. Once a significant relationship was found between each of the variables, we proceeded to estimate the model and its fit with indices and residuals.

Normality. We proceeded to estimate the normal distribution with the kurtosis parameter multivariable assuming that a value less than five is evidence of normality and the statistical sampling and significance Bootstrap with a value close to zero.

Reliability. We estimated the internal consistency with the correlation item subscale for which an alpha value greater than 0, 60 and lower 
than 0,90 was assumed as evidence of reliability. The reagents that reduced the required threshold were rejected. In this way, the eight factors obtained alphas between 0.60 and 0.75 , which were considered as enough evidence of internal consistency.

Validity. The Kayser Meyer Olkin (KMO) parameters and the Bartlet test were weighted to establish adequacy and sphericity while the item factor correlation was based on an Exploratory Factor Analysis of principal components with varimax rotation. it was considered as evidence of construct validity if the value was greater than 0,300

Structure. An analysis of structural equations was carried out with the factors used and the ethical hypothesis relations of dependence. The below 0,90 and above 0,40 values were considered as evidence of relationship whereas values near zero were taken as spurious relationships. In contrast, values higher than 0,90 were considered as evidence of collinearity and multicollinearity.

Adjustment. Hypothesis testing was performed using the chi square whose value and significance level was near zero rum taken as evidence s acceptance of the null hypothesis. On the contrary, values higher than 0.05 were considered as evidence of acceptance of the alternative hypothesis. However, because the sample was 210 students, the chi square parameter proved to be sensitive to the sample size. This was how the Adjustment Goodness Index (GFI) and the Mean Quadratic Approximation Error (RMSEA) were included.

\section{Results}

The table shows eight factors. The first included the items R22, R23 and R24 relative to values which explained $21 \%$ of the variance. The second factor referred to beliefs included reagents R19, R20 and R21 explaining $18 \%$ of the variance. The third perceptual factor included symptoms R16, R17 and R18 explaining 15\% of the variance. The fourth factor relating to motives included items R13, R14 and R15 explaining $13 \%$ of the variance. The fifth attitudinal factor included reagents R10, R11 and R12 explaining $11 \%$ of the variance. The sixth normative factor included indicators R7, R8 and R9 explaining $8 \%$ of the variance. The seventh factor included the items R4, R5 and R6 explaining $6 \%$ of the variance and the eighth experiential factor included the reactants R1, R2 and R3 explaining $3 \%$ of the variance.

\begin{tabular}{|c|c|c|c|c|c|c|c|c|c|c|c|c|}
\hline $\mathbf{R}$ & M & S & $\mathbf{K}$ & $\mathbf{A}$ & F1 & F2 & F3 & F4 & F5 & F6 & F7 & F8 \\
\hline R1 & 3.01 & 0.82 & 1,32 & ,769 & & & & & & & & 436 \\
\hline $\mathbf{R 2}$ & 2.93 & 0.73 & 1,43 &, 763 & & & & & & & &, 534 \\
\hline $\mathbf{R 3}$ & 2.81 & 0.71 & 1,50 & ,761 & & & & & & & &, 541 \\
\hline R4 & 2.71 & 0.82 & 1,87 &, 783 & & & & & & & ,453 & \\
\hline R5 & 3.71 & $\begin{array}{l}0.39 \\
\end{array}$ & 1,15 &, 783 & & & & & & &, 549 & \\
\hline R6 & 2.71 & 0.46 & 1,14 &, 702 & & & & & & & 457 & \\
\hline R7 & 2.81 & 0.31 & 1,13 &, 784 & & & & & & ,541 & & \\
\hline $\mathbf{R 8}$ & 1.71 & 0.37 & 1,15 &, 743 & & & & & & 563 & & \\
\hline R9 & 1,27 & 0.36 & 1,16 &, 715 & & & & & & ,670 & & \\
\hline R10 & 1.39 & 0.82 & 1,18 & 762 & & & & & ,675 & & & \\
\hline R11 & 1.01 & 0.81 & 1,19 & ,778 & & & & &, 561 & & & \\
\hline R12 & 1.72 & 0.93 & 1,10 &, 704 & & & & & ,674 & & & \\
\hline R13 & 1.42 & 0.49 & 1,18 & ,781 & & & & 672, & & & & \\
\hline R14 & 1.57 & 0.57 & 1,71 &, 734 & & & & 457 & & & & \\
\hline R15 & 3.81 & 0.71 & 1,16 & ,761 & & & &, 435 & & & & \\
\hline R16 & 2.31 & 0.29 & 1,15 & ,791 & & & ,541 & & & & & \\
\hline R17 & 1.82 & 0.49 & 1,16 &, 763 & & &, 549 & & & & & \\
\hline R18 & 3.49 & 0.57 & 1,17 &, 743 & & & 457 & & & & & \\
\hline R19 & 2.37 & 0.72 & 1,19 & ,761 & & 671 & & & & & & \\
\hline R20 & 2.81 & 0.61 & 1,01 &, 762 & & ,643 & & & & & & \\
\hline R21 & 1.80 & 0.83 & 1,91 &, 761 & & 560 & & & & & & \\
\hline R22 & 1.92 & 0.71 & 1,32 &, 776 &, 541 & & & & & & & \\
\hline $\mathbf{R 2 3}$ & 3.14 & 0.87 & 1,14 &, 794 & 456 & & & & & & & \\
\hline R24 & 2.93 & 0.77 & 1,16 &, 763 & 432 & & & & & & & \\
\hline
\end{tabular}

Note: Elaborated with data study: $R=$ Reactive, $M M=$ Median, $S=$ Standard Deviation, $K=$ Kurtosis, A = Alpha removed value item. Kurtosis Multivariable $=2,394 ; \mathrm{KMO}=0.719 ; \mathrm{X} 2=3.719,15 \mathrm{gl}, \mathrm{p}=0.000, \mathrm{~F} 1=$ Values $(21 \%$ explained total variance), $\mathrm{F} 2=\mathrm{Beliefs}(18 \%$ explained total variance), $F 3=$ Perceptions ( $15 \%$ explained total variance), $F 4=$ Reasons $(13 \%$ explained total variance), F5 = Attitudes $(11 \%$ explained total variance), $F 6=$ Norms ( $8 \%$ explained total variance), $F 7=$ Intentions $(6 \%$ explained total variance); $F 8=$ Experiences $(3 \%$ explained total variance). Table 1: Normal distribution, reliability and validity

Once the factors that explained $93 \%$ of the total variance were established, we proceeded to estimate their linear relationships from the correlations and covariances between them in order to observe their structure of trajectories that determine abortion experiences (see Table 2). 


\begin{tabular}{|c|c|c|c|c|c|c|c|c|c|c|c|c|c|c|c|c|}
\hline & F1 & F2 & F3 & F4 & F5 & F6 & F7 & F8 & F1 & F2 & F3 & F4 & F5 & F6 & F7 & F8 \\
\hline F1 & 1,0 & ,65* &, $61^{* *}$ &, $67 *$ & ,60** & $68 * * *$ & ,54* &, $62 *$ & 1,9 & ,54 & ,53 & ,50 & ,57 & $\begin{array}{l}, 58 \\
\end{array}$ & ,56 &, 67 \\
\hline F2 & & 1,0 & ,54* & ,52* & ,67* & „67* & ,60* & $59 * * *$ & & 1,8 & ,51 & 64 & 69 & $\begin{array}{l}, 59 \\
\end{array}$ & ,67 & 68 \\
\hline F3 & & & 1,0 & ,54* & $63^{* * *}$ & $66^{* *}$ & ,62* &, $51 * *$ & & & 1,7 & ,67 & ,68 & ,56 & ,66 & ,64 \\
\hline F4 & & & & 1,0 & ,64** &, $59 * *$ & ,64* & ,54* & & & & 1,8 & ,62 & ,49 & ,52 & ,58 \\
\hline F5 & & & & & 1,0 & ,53* & $61 * *$ & ,57* & & & & & 1,8 & ,51 & ,51 & ,65 \\
\hline F6 & & & & & & 1,0 &, $56^{*}$ &, $60^{* * *}$ & & & & & & 1,9 & 69 &, 60 \\
\hline F7 & & & & & & & 1,0 & ,62* & & & & & & & 1,7 & ,48 \\
\hline F8 & & & & & & & & 1,0 & & & & & & & & 1,6 \\
\hline
\end{tabular}

Note: Elaborated with data study: F1 = Values, F2 = Beliefs, F3 = Perceptions, F4 = Reasons, F5 = Attitudes, F6 = Norms, F7 = Intentions; F8 = Experiences: $* p<, 01 ; * * p<, 001 ; * * * p<, 0001$

Table 2: Correlations and covariances

An analysis of structural equations with maximum likelihood was carried out in which the experiences related to the request for induced abortion were predicted by the group norms followed by the intentions and the perceptions.
As family norms intensify, experiences of requesting termination of pregnancy increase, although decisions to perform behaviors associated with the request for abortion also increase, but perceptions of risk associated with the abortion experience also increase they increase (see Figure 1).

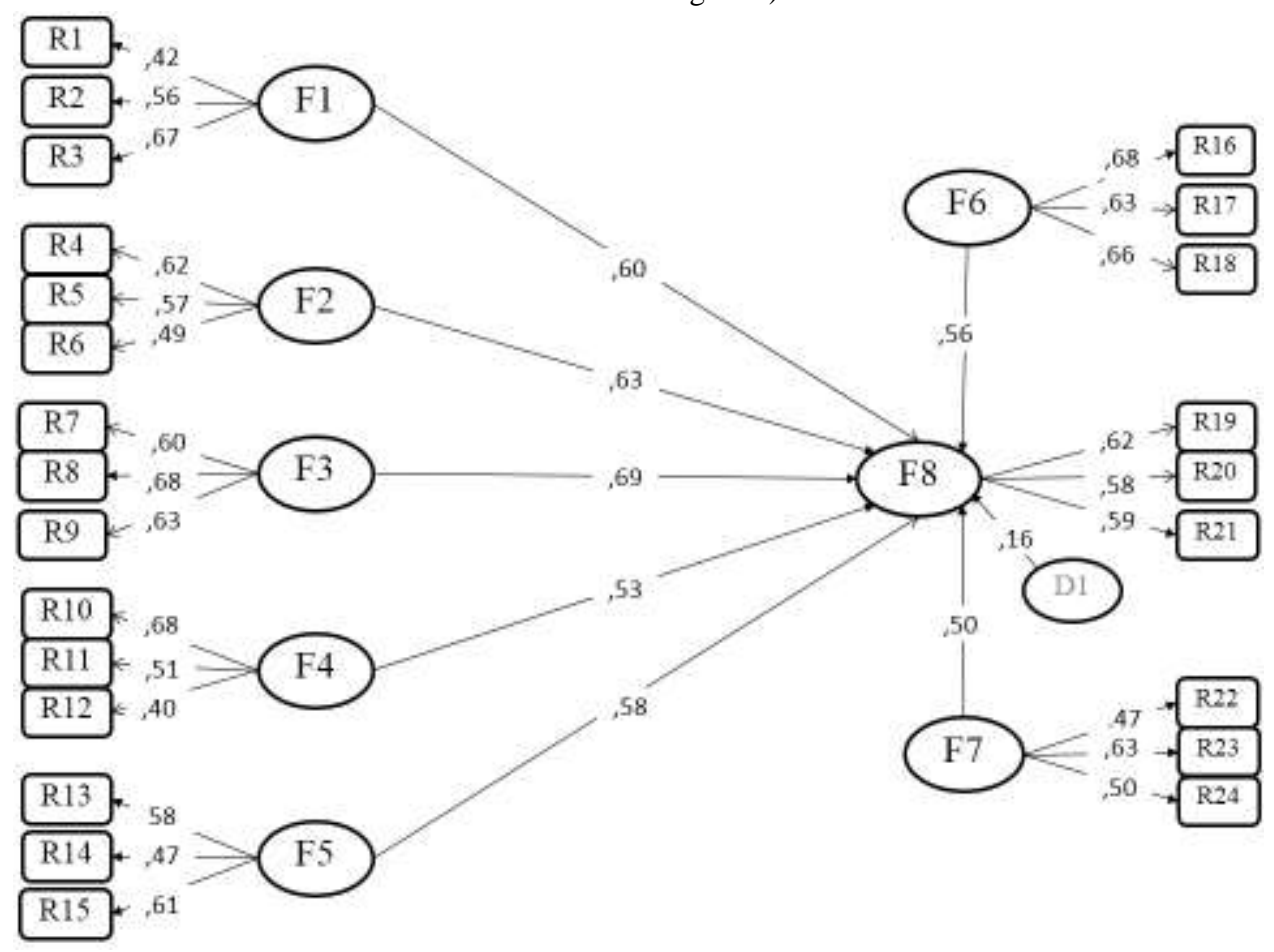

Note: Elaborated with data study: F1 = Values, F2 = Beliefs, F3 = Perceptions, F4 = Reasons, F5 = Attitudes, F6 = Norms, F7 = Intentions; F8 = Experiences:

Figure 1: Structural equation modelling

On the other hand, the square chi value $\left(\mathrm{X}^{2}=17.829,21 \mathrm{gl}, \mathrm{p}=0.001\right)$, the Adjustment Goodness Index $(\mathrm{GFI}=0.950)$ and the Mean Quadratic Approximation Error (RMSEA $=0.003$ ) show the acceptance of the null hypothesis relative to the dependency relations among the eight factors used.

\section{Discussion}

In reference to the studies carried out by the state of the matter, the present work has established dependency relations between the variables and exposed factors.
Regarding the studies that reported a series of beliefs, attitudes and knowledge of contraception that were not enough to avoid unwanted pregnancies and the eventual request or practice of curettage, the present investigation warns that it is the influence of family and friends who affect to a greater extent on abortion decisions. In the case of family support, it is considered a social support for adherence to induced abortion.

However, the experience of a uterine curettage is far from requesting information, supporting an abortion practitioner, attending an expert conference or dissemination talks. It is recommended to carry out uterine curettage interviews to establish relationships with their values, norms, 
perceptions, beliefs, attitudes, intentions and experiences prior to the practice itself.

It should be noted that the results are only representative of the nonprobabilistic sample of 210 students. In this sense, it is recommended to complement the results with other diagnoses in health professionals, family members and couples since medical malpractice or negligence is a factor of death that spreads in the perception of risk. On the other hand, the influence of the family infiltrates the group norms of the applicants and / or abortion practitioners. Finally, when influencing the type of relationship in abortion decisions, it is essential to study the perceptions that couples have about the relationship with the applicants and abortion practitioners.

The application of the results to university health programs is fundamental given the percentage of the student population that is forced to interrupt their studies to address parental responsibility or the social and family consequences of the abortion practice. It is recommended to intervene in families and groups of friends in order to identify the priorities of the applicants or abortion practitioners in the context of their university studies and / or the exercise of their profession.

\section{Conclusion}

The present study has established relationships of dependence between the group norms and the experiences related to the request of induced abortion in a public university context. These findings allow to anticipate the tendency of university students around their sexuality, request and eventual induced abortion. However, decisions to be influenced by family support or the absence of a group of belonging or reference, it is essential to consider the incidence of religious groups in unwanted pregnancies and their eventual abortion request or practice as this means that decisions they are emotional strings.

\section{References}

1. Calderón, J \& Alzamora, L (2009). Couple and group factors related to induced abortion in adolescent women. Scientia, 1, 110-116.

2. Chávez, M \& Zapata, J (2009). Attitudes regarding sexuality in university students. Teaching and Research in Psychology, 14, 137-151.

3. Fernandez, M. Carr, F Leal, M Carrillo, Carrillo I, E Lozano, Fernandez M, S \& Pastor, L (2010). An approach to abortion and immigration in Spain. The socio-economic value of human life. Cuadernos de Bioética, 21, 313-327

4. Galváo, L Díaz, J Osis, M C lark, S \& Ellerston, C (2000) Emergency contraception: knowledge, attitudes and practices of gynecologists. International Perspectives in Family Planning. Special number; 1-5

5. González, H (2000). Theoretical aspects for the sociodemographic study of adolescent pregnancy. Northern Border; 12, 65-85

6. Obeichina, J Mbamara, U Uboataja, O Ogelle, O \& Akabuike, C (2010). Knowledge, attitude and practice of emergency contraception among studies in tertiary schools in Anambra
State Southeast Nigeria.International Journal in Medicine and Medical Science, 2, 1-4

7. Oduwole, E (2010). Personhood and abortion: an African perspective. Lumina; 21, 1-10

8. Olaitan, O (2011). Attitudes University studies toward abortion in

Nigeria. Journal of Neuroscience and Behavioral Health; 3, 7469

9. Petracci, M (2011). Opinions and social representations of men about abortion in Buenos Aires, Argentina. Sexuality, Health and Society; 8, 10-35

10. Piaroza, R Kazembe, A Maluwa, A C Hirwa, E \& Chimango, J (2012). Psychological distress among adolescents before and after unsafe induced abortion in Malawi. Journal of Research in Nursing and Midwifery; 2, 29-36

11. Ramírez, C (2000). Knowledge and attitudes towards sexuality in secondary school teachers. Sonorense Magazine of Psychology; 13, 47-54

12. Rodríguez, G \& Mayol, V (2011). Attitude of adolescent men before pregnancy in adolescence. Evidences in Pediatrics; 7, 14

13. Salazar, H (2007). Behaviors, knowledge and attitudes of adolescent students in urban areas of cities against contraceptive methods. Journal of Psychology Herediana; 2, $34-44$

14. Serrano, M (2011). Knowledge, attitude and perception of resident gynecology and obstetrics doctors of ISSSTE about emergency

contraception. Specialties magazine Doctor Surgical; 16, 215 220

15. Shelat, P Hihoriya, M \& Kumbar, S (2012). Knowledge and attitude towards the use of emergency contraceptive pills among college students. International Journal of Basic \& Clinical Pharmacology; 1, 77-84

16. Silva, M Ashton, $\mathrm{T} \& \mathrm{McNeil,} \mathrm{R}$ (2011). Improving termination of pregnancy services in New Zealand. New Zealand Medical Journal; 124, 83-90

17. Sultan, A \& Malik, R (2010). Emergency contraceptive pills: exploring to knowledge and attitudes of community health workers in a developing Muslim country. North American Journal of Medical Sciences, 2, 359-364

18. Tapia, A Villaseñor, M \& Nuño, B (2008). Knowledge and attitudes towards the use of emergency contraception in university students. Medical Journal of the Mexican Institute of Social Security; 46, 33-41

19. Távara, L \& Sacsa, D (2008). Knowledge attitudes and practices of obstetrician gynecologists in relation to abortion. Lima: Promsex.

20. Viveros, M \& Navia, (2012). The place of masculinities in the decision of abortion. Sexuality, Health and Society; 12, 135163 
This work is licensed under Creative Commons Attribution 4.0 License

To Submit Your Article Click Here: Submit Manuscript

DOI:10.31579/2578-8868/174
Ready to submit your research? Choose Auctores and benefit from:

* fast, convenient online submission

* rigorous peer review by experienced research in your field

* rapid publication on acceptance

* authors retain copyrights

* unique DOI for all articles

* immediate, unrestricted online access

At Auctores, research is always in progress.

Learn more www.auctoresonline.org/journals/neuroscience-andneurological-surgery 